Mitteilungen der Österreichischen Geographischen Gesellschaft, 162. Jg., S. 550-555

(Annals of the Austrian Geographical Society, Vol. 162, pp. 550-555)

Wien (Vienna) 2020, https://doi.org/10.1553/moegg162s550

\title{
Heinz SLupetzky zUM ACHTZiger
}

\author{
Gerhard Karl LIEB, Graz*
}

mit 2 Abb. im Text

\section{Eine persönliche Einleitung}

Am 17. Jänner 2020 vollendete Heinz SLuPETZKy sein 80. Lebensjahr, was seine akademische Heimstätte, die Universität Salzburg, mit einer Festveranstaltung eine Woche später würdigte. Es war mir nicht möglich, an dieser teilzunehmen, da ich zuvor bereits einen Vortrag auf einer interdisziplinären Tagung zu Klimawandel am selben Tag zugesagt hatte. Der Zufall wollte es, dass das Thema meines Vortrags „Österreichs Hochgebirge im Wandel - Veränderungen von Gletschern und anderen Landschaftselementen" genau jene Interessenfelder umfasste, die Heinz SLuPETZKY sein Leben lang beschäftigt haben - und zu denen er auch mir wertvolle Impulse gegeben hat. Gerne nehme ich daher die Gelegenheit wahr, ihm mit dieser kleinen Gratulationsschrift hierfür zu danken.

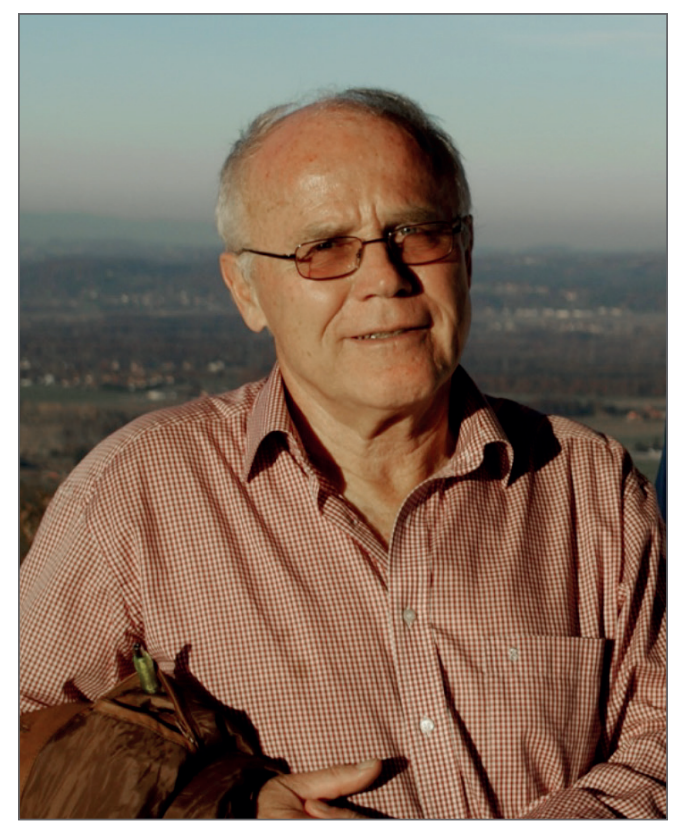

Heinz SluPETZKY (Foto: G. K. Lieb 2010)

\footnotetext{
* Ao. Univ.-Prof. Mag. Dr. Gerhard Karl Lieb, Universität Graz, Institut für Geographie und Raumforschung, Heinrichstraße 36, A-8010 Graz. - E-Mail: gerhard.lieb@uni-graz.at.
} 
Als ich mich im Zuge meines Geographiestudiums ab etwa 1980 der Hochgebirgs- und Gletscherforschung zuzuwenden begann, kam mir schon bald über die Vermittlung meines Dissertationsvaters Herwig WaKONIGg der Name Heinz SLupeTZKY in der Konnotation als der österreichische „Gletscherpapst“ unter. Schon bald kreuzten sich unsere Wege erstmals auf einer Veranstaltung in „seinem“ Stubachtal (siehe unten). Ab den späten 1980er Jahren suchte ich, inzwischen Postdoc im Habilitationsstadium, wegen seiner Fachexpertise wiederholt seinen Rat, woraus bald erste gemeinsame Geländearbeiten im Hochgebirge und eine gemeinsame Publikation entstanden. Heinz SLUPETZKY hat dann auch ganz wesentlich mein Habilitationsverfahren unterstützt, und aus dieser intensivierten und produktiven Kooperation entstand schließlich eine bis heute währende Freundschaft.

Die folgenden Zeilen bieten weder eine vollständige biographische Darstellung noch eine umfassende Würdigung seiner wissenschaftlichen Leistungen, die ich mir im vollen Umfang gar nicht zutrauen dürfte. Hierfür kann vielmehr auf die Biographie von H. HeUberger in der Festschrift zum 60. Geburtstag von Heinz SlupetzKy (AschenkrenNer und Suida 2000, S. 1-6) sowie auf das wertschätzende Vorwort von H. Riedl zu SLupetzKy (2020) verwiesen werden. Stattdessen möchte ich versuchen, das Schaffen von Heinz SLUPETZKY thematisch zu umreißen und in einen größeren Kontext zu stellen. Dabei gehe ich kursorisch und exemplarisch vor und muss mit Nachdruck darauf hinweisen, dass meine persönliche Darstellung nur Teilaspekte des Werkes eines der Großen der österreichischen Physiogeographie der Gegenwart beleuchten kann.

\section{Gletscherforschung in Langzeitperspektive}

Von den oben genannten Themen assoziiert man Heinz SLUPETZKY zu Recht in allererster Linie mit Gletschern. Seine entsprechenden Arbeiten gehen auf das Jahr 1960 zurück, als die Zwillingsbrüder Heinz und Werner im inneren Stubachtal (im heutigen Salzburger Anteil des Nationalparks Hohe Tauern) Gletschermessmarken anlegten. Diese begründeten eine seit damals durchgehende Messreihe, die sich noch heute durch jährliche Nachmessungen im Rahmen des Gletschermessdienstes des Österreichischen Alpenvereins erweitert. Heinz SLUPETZKY blieb durch seine Dissertation dem Stubachtal treu und entwickelte dieses in wenigen Jahren zu einer - wie man heute sagen würde - hochwertigen „monitoring site“ nicht nur für Veränderungen der Gletscher, sondern auch der steuernden Faktorengruppe Klimaelemente und der nachgeordneten hydrologischen Komponenten. „Hochwertig“ bezieht sich einerseits auf die Methodik, worin die Aktivitäten stets auf der Höhe der Zeit oder auch dieser voraus waren, und andererseits auf die in verschiedenen Ebenen erfolgte Einbindung in nationale und internationale Forschungsnetzwerke, in denen das Stubachtal jeweils zu den tragenden Säulen sowohl in Bezug auf die Verfügbarkeit als auch die Qualität der Daten gehörte.

Zu Methodik und Datenqualität seien beispielhaft zwei besondere Merkmale in der Arbeitsweise von Heinz SLuPETZKY herausgegriffen. Das eine ist sein lebenslanger Schwerpunkt auf Geländearbeit, das heißt bei ihm, insbesondere Messungen direkt am, auf, auch unter dem Gletscher, inklusive beinharter Knochenarbeit beim Transport der Geräte, beim Spuren im hüfttiefen Schnee oder beim Graben von Schneeschächten - und das eingebettet in wochenlange Aufenthalte vor Ort, um den jeweils besten Zeitpunkt zu erwischen, etwa um die maximale Ausaperung zu dokumentieren. Gewissermaßen nebenbei begann er, wie andere Gletscherforscher auch, seine Objekte fotografisch zu dokumentieren, entwickelte dabei aber eine professionelle Meisterschaft, in der sich auch seine künstlerische Begabung manifestiert (die er übrigens auch außerhalb der Fotografie lebt). Das zweite Merkmal ist sein Bestreben, in Kooperation mit Einzelpersonen und Institutionen hochgenaue Karten ,seiner“ Gletscher zu entwickeln, was sich in einer beeindruckenden Zahl an 
solchen, unter seiner Ägide entstandenen Produkten äußert. In die Kartengestaltung floss stets seine unvergleichbare Gebietskenntnis, gepaart mit seinem exakten Blick für Details ein und letztlich entstand in der Kombination aus Orthofoto und Strichkarte sowohl ein Alleinstellungsmerkmal von hohem Wiedererkennungswert als auch ein beispielloser kartographischer Standard.

Ein weiteres herausragendes Merkmal der Gletscherforschung „Marke Slupetzky“ war es, diese auf Langfristigkeit auszurichten. Im Kontext des modernen Klimawandeldiskurses sind Langzeitreihen von Klimaelementen und von damit im Zusammenhang stehenden Größen, die Klimawandelfolgen quantifizierbar machen, die wohl wichtigsten Grundlagen. Dabei wird in der (auch wissenschaftlichen) Öffentlichkeit nur allzu oft übersehen, was es heißt, Messnetze langfristig konzeptionell, finanziell, organisatorisch und personell - zu sichern und damit erst seriöse Daten über die in Rede stehenden Veränderungen zu erhalten. Hier hat Heinz SLuPETZKy Einzigartiges geleistet und über sechs Jahrzehnte selbst Hand angelegt. Die Massenbilanzreihe vom Stubacher Sonnblickkees sei hierbei als eine der weltweit längsten und geschlossensten hervorgehoben!

\section{Vom Stubachtal in die Welt}

Mit dieser Langfristigkeit notwendigerweise verbunden ist aber auch ein gehöriges Maß an Ortsfestigkeit. Diese bezieht sich bei Heinz SLUPETZKy primär auf das innere Stubachtal, wo er seit den späten 1950er Jahren sprichwörtlich zu Hause ist. Dass es ausgerechnet dieses Tal wurde, war insofern Zufall, als SLUPETZKYs Eltern die Rudolfshütte zur Bewirtschaftung übernahmen. Die beiden bergbegeisterten Zwillingsbrüder Heinz und Werner erkannten aber sofort das wissenschaftliche Potenzial des geomorphologisch so vielgestaltigen und bis dahin glaziologisch so wenig erforschten Hochgebirgstals an der Nahtstelle der Glockner- und der Granatspitzgruppe und legten bereits 1960 aus Eigeninitiative Gletscher-Messmarken an. Diese wurden schon im Folgejahr in das Messprogramm des Alpenvereins aufgenommen, und Heinz SLUPETZKY blieb, wie schon erwähnt, mit seinem Dissertationsprojekt hier „hängen“. Der Titel der (bei Hans SPREITZER an der Universität Wien 1968 abgeschlossenen) Dissertation lautete „Glaziologische und glazialmorphologische Untersuchungen im obersten Stubachtal (Hohe Tauern), mit besonderer Berücksichtigung der Massenhaushaltsuntersuchungen am Stubacher Sonnblickkees in den Jahren 1963-1966“.

Damit ist erneut der Name eines jener beiden Gletscher gefallen, die durch Heinz SLUPETZKY zu den am besten erforschten der gesamten Alpen werden sollten: Während das Sonnblickkees (siehe die Abbildung) als Hanggletscher eine Bergflanke bedeckt, bildet das Ödenwinkelkees eine stark schuttbedeckte Gletscherzunge aus, die aus einem von hohen Felswänden umgebenen Kessel herausfließt.

Wie Langfristigkeit und Ortstreue in idealer Weise nicht nur einfach lange Datenreihen entstehen lassen, sondern auch zu wissenschaftlichen Erkenntnissen führen können, die ohne diese Kombination nicht möglich gewesen wären, kann man der monographischen Arbeit, die anlässlich seines 80. Geburtstages erschienen ist, entnehmen (SLUPETZKY 2020). Darin zeigt er nicht nur die wertvollen langen Reihen, sondern es gelingt ihm, durch umsichtige Interpretation langjähriger, akribisch gesammelter und dokumentierter Detailbeobachtungen das bisher noch weitgehend unbekannte (oder fehlinterpretierte) geomorphologische Phänomen von „Schuttnetzwerken“ („,debris ridge networks") proglazialer Gebiete exakt zu beschreiben und zu deuten.

Ungeachtet seiner sprichwörtlichen „Treue“ zum Stubachtal hat Heinz SLUPETZKY aber selbstverständlich auch in anderen Gebieten geforscht. Auch hierbei versuche ich keine vollständige Aufzählung, sondern beschränke mich auf schlaglichtartige Erwähnungen: So ist seine Affinität zu den Hohen Tauern, die im letzten Abschnitt dieses Textes noch eine Rolle spielen wird, unverkennbar, besonders durch Arbeiten am Obersulzbachkees und an der Pasterze, wo ich jeweils auch mit ihm 


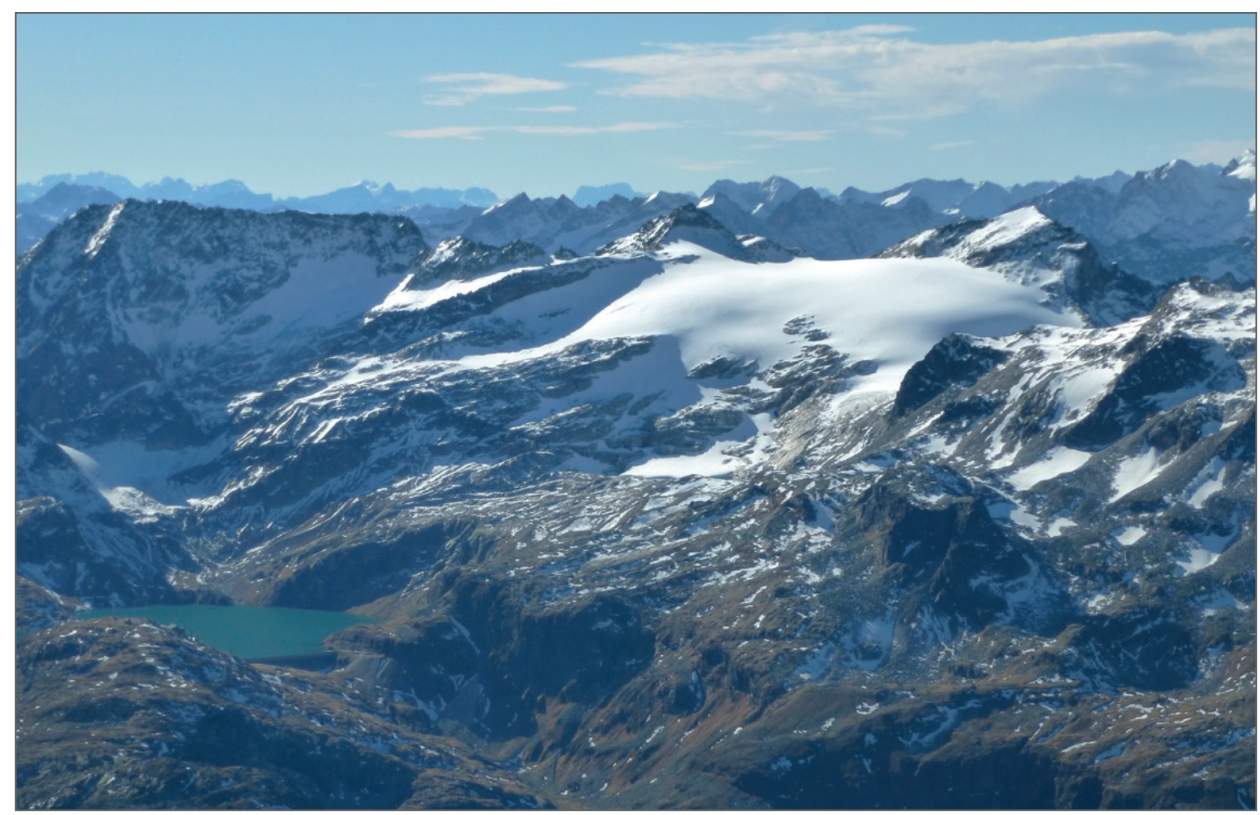

Das Stubacher Sonnblickkees im inneren Stubachtal (Hohe Tauern, Salzburg), ein zentrales Forschungsgebiet von Heinz SLupeTzKY, gesehen vom Gipfel des Kitzsteinhorns nach Südwesten (Foto: G. K. Lieb 2013)

zusammenarbeiten durfte. Außerdem darf Heinz SLUPETZKY auf physiogeographischem Gebiet auch weit über den Fokus auf Gletscher und sein Habilitationsfach Hydrogeographie hinaus - als einer der besten Kenner seiner Wahlheimat Salzburg gelten, was sich in zahlreichen, thematisch breit gestreuten Publikationen widerspiegelt. Ein besonderes Erlebnis ist es, wenn man eines der Geotope von ihm selbst gezeigt bekommt und dabei sowohl an seinem vernetzenden Wissen teilhaben als auch sich von seiner Begeisterung anstecken lassen kann!

Aus den Forschungsnetzwerken, in die Heinz SLUPETZKY schon so früh eingebunden war, erwuchsen aber auch zahlreiche internationale Aktivitäten - von einer Gastprofessur in den USA bereits in den 1970er Jahren bis hin zu seinen Forschungen auf Franz-Josef-Land, die er als einer der ersten, unmittelbar nachdem dessen Status als militärisches Sperrgebiet der Sowjetunion Anfang der 1990er Jahre aufgehoben worden war, durchführte. Ein bezeichnendes Beispiel für seinen akribischen, aus dem Stubachtal in die Welt getragenen Arbeitsstil ist die Bearbeitung eines kleinen Kargletschers im Grenzgebiet von Kanada und Alaska - mit exakter Gletscherkarte und Interpretationen, die eindrucksvoll zeigen, wie Heinz SLUPETZKy über die lokalen Fallstudien hinaus stets das große Ganze seines Fachgebietes im Blick hat.

\section{Gelebte Verantwortung für Gesellschaft und Umwelt}

Dieses große Ganze meint aber nicht bloß die Geographie, sondern hat für Heinz SLuPETZKy stets auch deren gesellschaftliche Relevanz bedeutet. Hierbei durchziehen zwei Aspekte sein Schaffen: Zum einen lassen seine Kooperationen und Publikationen schon sehr früh das Bemühen erken- 
nen, Wissen nicht um seiner selbst willen zu generieren, sondern im Sinne angewandter Wissenschaft gesellschaftlichen Bedarf nach wissenschaftlicher Expertise zu befriedigen. So waren seine Arbeiten stets, wie erwähnt, eingebunden in praxisorientierte Forschungsnetzwerke, speziell mit wasserwirtschaftlichem Hintergrund. Darüber hinaus war er aber auch unmittelbar in die Lösung praktischer Probleme aus seinen Wissensgebieten involviert, etwa als Gutachter bei Themen mit Bezug zu Gletschern, Wasser, Schnee, Eis und Lawinen. Zum anderen war und ist es ihm stets ein Anliegen, das von ihm und seinen Teams generierte Wissen einer breiten Öffentlichkeit zugänglich zu machen. Daraus ergibt sich ein umfangreicher Schwerpunkt in seinem Arbeits-Oeuvre, der dem Bereich Wissenschaftspropädeutik bzw. der Landschafts- und Umweltdidaktik zugeschrieben werden kann. Ergebnisse davon sind zahlreiche - meist federführende - Mitwirkungen an Projekten der Natur- und Umweltbildung, von der Lehrtafel über die vollständige Konzeption von Themenwegen bis hin zu einschlägigen Buchprojekten.

Es sei mir gestattet, aus den Buchprojekten ein von mir gemeinsam mit Heinz SLUPETZKY realisiertes herauszugreifen, weil es die Einbettung seiner Bemühungen in ein weites gesellschaftliches Umfeld illustriert. Es handelt sich um eine Monographie über den größten Gletscher Österreichs, die Pasterze (LieB und SLUPETZKY 2011). Zu deren Erstellung bekamen wir den Auftrag anlässlich des 30-jährigen Jubiläums der Gründung des Nationalparks Hohe Tauern (und damit des Bestehens von Nationalparken in Österreich), und wir bemühten uns darin um eine integrativ-geographische Narration weit über Gletscher hinaus. Auftrag- und Herausgeber waren zwei Organisationen, mit denen Heinz SLuPETZKY in besonderer Weise verbunden war und ist: (1) Der Österreichische Alpenverein spielte schon zu Beginn seines wissenschaftlichen Werdegangs eine Rolle, war es doch letztlich dieser Verein, über dessen „Vermittlung“ seine Eltern ins Stubachtal kamen, er seine frühe alpinistische Sozialisation erhielt und in Fragen der alpinistischen Sicherheit im Laufe der Zeit zu einer gerne zu Rate gezogenen „Instanz“ wurde (z. B. WüRTL 2018). (2) Der Nationalpark Hohe Tauern kam später dazu, denn als die Idee zu dessen Realisierung 1971 in Form der Dreiländervereinbarung von Heiligenblut erstmals politisch konkret wurde, war Heinz SLUPETZKY im Stubachtal als einem späteren Teilgebiet schon ein gutes Jahrzehnt so etwas wie eine fixe Größe, die als Person dort einfach dazugehörte. Die Nationalparkidee scheint ihn von Anfang an begeistert zu haben, und so verwundert es nicht, dass er den Nationalpark Hohe Tauern später in vielfältiger Weise unterstützte und seine Expertise in dessen Dienst stellte.

Sich für Nationalpark und Alpenverein einzusetzen, ist aber auch ein politisches Statement, und damit komme ich zu einer letzten Facette, die ich an Heinz SLuPETzKy hervorheben möchte. Es geht um Betroffenheit und um Verantwortung für Gesellschaft und Umwelt, die er auf vielfältige Weise übernahm, sei es durch seine Tätigkeit in der universitären Selbstverwaltung, in Beiräten zum Nationalpark oder als Funktionär im Alpenverein, dessen großer Sektion Salzburg er von 2000 bis 2010 vorstand. Diese aufopfernde, ehrenamtliche Tätigkeit scheint übrigens bei den SLUPETZKYs in der Familie zu liegen - auch Brigitte, seine Frau, und Nicole, eine von zwei Töchtern der beiden, sind in hochrangigen Funktionen für den Alpenverein aktiv. Jedenfalls ersieht man daran - und somit möchte ich den Bogen wieder zurück zur Wissenschaft spannen -, dass Heinz SLUPETZKY stets bereit gewesen ist, sich für das, was aus einer ethischen Perspektive hinter der Beschäftigung mit seinen Forschungsobjekten steht, öffentlich einzusetzen, nämlich für den Natur- und Klimaschutz sowie für die nachhaltige Entwicklung der Alpen.

Damit ist Heinz SLUPETZKY das nachahmenswerte Beispiel eines Wissenschaftlers, der nicht nur auf akademischem Boden seine Pflichten in Lehre, Forschung und Verwaltung (über)erfüllt, sondern weit darüber hinaus zum Gemeinwohl beigetragen hat. Dafür - und für seine Vorbildfunktion in Bezug auf Neugier, Akribie, Genauigkeit und Engagement - gebührt ihm der Dank von seinen Studierenden, Kolleginnen und Kollegen sowie allen, die ein Stück des Weges mit ihm gemeinsam gehen durften. 


\section{Literaturverweise im Text}

Aschenbrenner J., Suida H. (Hrsg.) (2000): 1960-2000. 40 Jahre glaziologische Forschung. Festschrift für Heinz Slupetzky zum 60. Geburtstag. Salzburg: Selbstverlag des Fachbereichs Geographie und Geologie der Universität Salzburg, 215 S. (= Salzburger Geographische Arbeiten, 36).

Lieb G. K., Slupetzky H. (2011): Die Pasterze. Der Gletscher am Großglockner. Salzburg: Pustet Verlag, $159 \mathrm{~S}$.

SluPETZKy H. (2020): Das Ödenwinkel- und Riffelkees und die Entstehung von Schuttnetzwerken in den Gletschervorfeldern (Stubachtal, Hohe Tauern). Eine Dokumentation über 60 Jahre Forschung. Salzburg: Selbstverlag des Fachbereichs Geographie und Geologie der Universität Salzburg, 109 S. (= Salzburger Geographische Arbeiten, 49).

WÜRTL W. (2018): Heinz Slupetzky - Alpinexperte, Wissenschaftler, Funktionär, ... In: bergundsteigen (Internationale Zeitschrift für Sicherheit \& Risiko im Bergsport), 103, S. $26-31$. 